\title{
Minimizing light reflection from dielectric textured surfaces
}

\author{
Alexei Deinega, ${ }^{1, *}$ Ilya Valuev, ${ }^{2}$ Boris Potapkin, ${ }^{1}$ and Yurii Lozovik ${ }^{3}$ \\ ${ }^{1}$ Kinetic Technologies Ltd., Kurchatov Sq. 1, Moscow 123182, Russia \\ ${ }^{2} J o i n t$ Institute for High Temperatures RAS, Izhorskaya, 13, Bld. 2, Moscow 125412, Russia \\ ${ }^{3}$ Institute for Spectroscopy RAS, Fizicheskaya, 5, Troitsk, Moscow Region 142190, Russia \\ ${ }^{*}$ Corresponding author: poblizosti@kintech.ru
}

Received January 4, 2011; revised February 11, 2011; accepted February 15, 2011;

posted February 18, 2011 (Doc. ID 140551); published April 12, 2011

In this paper, we consider antireflective properties of textured surfaces for all texture size-to-wavelength ratios. Existence and location of the global reflection minimum with respect to geometrical parameters of the texture is a subject of our study. We also investigate asymptotic behavior of the reflection with the change of the texture geometry for the long and short wavelength limits. As a particular example, we consider silicon-textured surfaces used in solar cells technology. Most of our results are obtained with the help of the finite-difference time-domain (FDTD) method. We also use effective medium theory and geometric optics approximation for the limiting cases. The FDTD results for these limits are in agreement with the corresponding approximations. (c) 2011 Optical Society of America

OCIS codes: $\quad 310.1210,050.2770$.

\section{INTRODUCTION}

Elimination of undesired reflection from optical surfaces is important for many technologies, such as solar cell, lightsensitive detectors, lenses, displays, etc. To reduce reflection, one can use single-layer quarter-wave coatings [1], but their applications are limited by a small range of wavelengths and incidence angles. To extend this range, multilayer or gradient index coatings [2] can be used, however, their manufacturing encounters problems with thermal mismatch, adhesion, and stability of thin-film stacks [3].

An alternative method for the reflection reduction consists of texturing the surface with three-dimensional pyramids or two-dimensional grooves (gratings; Fig. 1) [4,5]. Many reports on successful fabrication of the antireflective textured surfaces have been published [3, $\underline{6}-\underline{9}$ ]. Their use in solar technology leads to significant enhancement of solar cell efficiency by reducing reflection from the surface of the cell by 1 or 2 orders of magnitude.

In the limiting cases of long and short wavelengths, this reduction in reflection can be explained theoretically with the help of effective medium and geometric optics approximations. According to the first approximation, if the wavelength is greater than the texture size, the texture behaves like a gradient index film with reduced reflection $[10,11]$. In the short wavelength limiting case, reflection reduction can be illustrated geometrically: rays should be reflected many times until being reverted back [12]. At the same time, transmitted rays deviate from the incident direction that leads to the light trapping effect used in solar cells [13]. It was also shown numerically that using texture reduces reflection for wavelengths comparable with its size. In particular, reflection decreases when texture is made higher and dielectric contrast between the texture and incident medium is lower $[14,15]$.

In our previous work, we studied antireflective properties of dielectric textured surfaces at the whole wavelength range, including long and short wavelength limits [16]. We found optimal parameters for the period of the texture and the pyramids' height. It was found that the key factor influencing the optimal pyramid size is the character of substrate tiling by the pyramid bases.

The present paper extends our previous work with new details and results. We closely analyze the limiting cases due to their importance for finding global reflection minimum at the whole wavelength range. It is demonstrated that the conclusions of this work are applicable to real silicontextured surfaces used in solar cell technology. In order to check the relevance of our calculations, we make a comparison with the experimental data.

As a main numerical tool, we use the finite-difference timedomain (FDTD) method [17]. This method implements direct numerical solution of Maxwell's equations, so it is valid for all texture size-to-wavelength ratios, including long and short wavelength limits. For accurate reflection calculation, it is important to reduce undesired numerical reflection from the artificial absorbing perfectly matched layer [17]. For this purpose, we use the additional backing absorbing layers technique that we described in [18]. We apply effective medium theory and ray tracing techniques for long and short wavelength limits as well. All calculations are performed using the free electromagnetic simulation package Electromagnetic Template Library [19]. It incorporates all three simulation methods (FDTD, effective medium theory, and ray tracing) and allows one to use them for the same geometry of the studied structure.

In the following, we consider surfaces coated by a periodic pyramid-type texture with height $d$. Pyramids bases have the shape of triangles, squares, hexagons, and circles (in the last case, the pyramid is, in fact, a cone) with the distance between the base side and its center $L$ (Fig. 1). The pyramids are closely packed on the substrate in the triangular or square lattice 


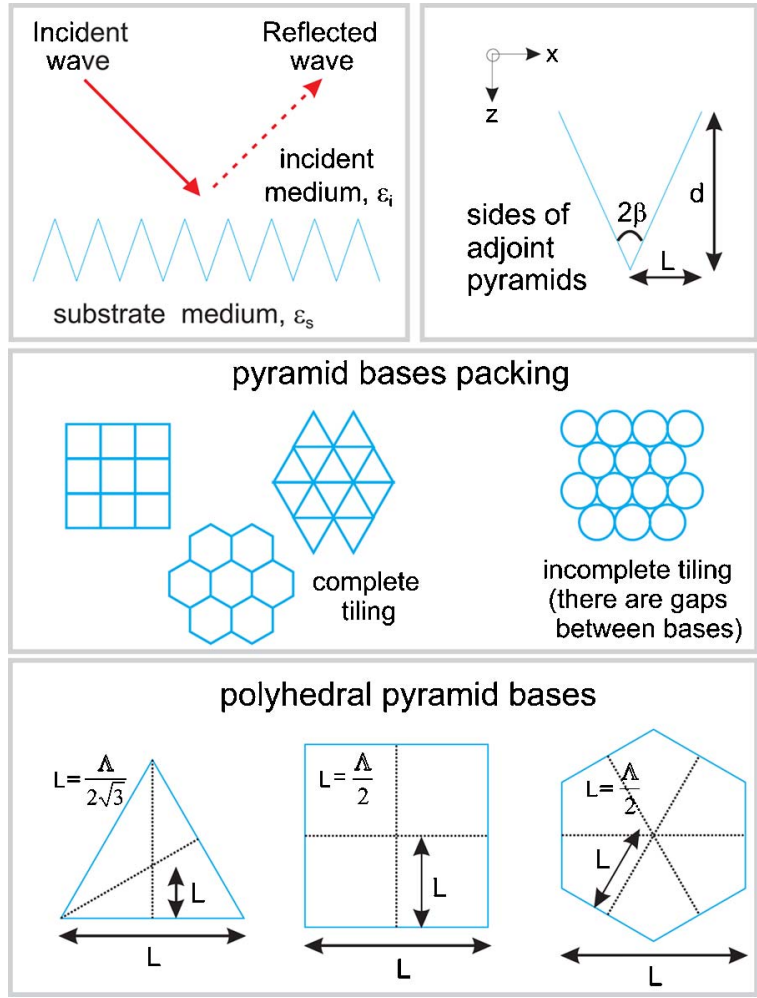

Fig. 1. (Color online) Antireflective textured surface: front and side view.

with the period $\Lambda$. In the following, we specially distinguish two cases (Fig. 1): complete tiling case when pyramids bases touch each other at every point of their perimeters (this corresponds to the polygon base pyramids in our study) and incomplete tiling case when there are gaps between bases (this corresponds to cones). Most of the results presented here are calculated for the normal light incidence case. However, as shown below, generalization to oblique incidence is possible.

The remaining paper is organized as follows. In Sections 2 and 3 , we estimate asymptotic behavior of the reflection with the increasing height-to-base size ratio for the pyramids for long and short wavelength limits. In Section $\underline{4}$, we consider antireflective properties of the textured surfaces at the whole range of their sizes and find the location of the global reflection minimum with respect to pyramid geometrical parameters. In these three sections, we study the nondispersive case considering glass-textured surfaces (the refractive index $n=1.5$ ). In Section $\underline{5}$, we present results for silicon-textured surfaces used in solar cell technology. In Section $\underline{6}$, we summarize our results.

\section{LONG WAVELENGTH LIMIT}

In the long wavelength limit $\Lambda \ll \lambda$, electromagnetic waves propagate in a heterogeneous structure as in an anisotropic medium of some effective (or homogenized) dielectric permittivity. Its value is determined by the shapes and the relative fractions of the structure components [20]. In our case, textured surface can be treated as a layer with gradually changing dielectric permittivity tensor $\hat{\varepsilon}(z)$ [11]. Here, the $z$ direction is aligned along the pyramid axis (see Fig. 2), with $z=0$ at the pyramid tops and $z=d$ at the pyramid bases. The $z$ component of $\hat{\varepsilon}(z)$ is the average [11]

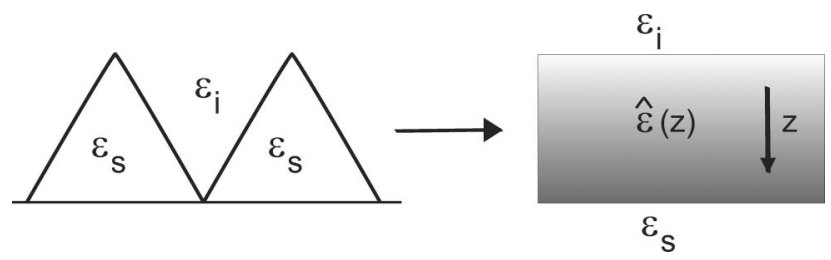

Fig. 2. In a long wavelength limit, textured surface can be treated as a layer with gradually changing dielectric permittivity tensor $\hat{\varepsilon}(z)$.

$$
\varepsilon_{z}(z)=f(z) \varepsilon_{s}+(1-f(z)) \varepsilon_{i}
$$

where $\varepsilon_{s}$ is the pyramid's permittivity, $\varepsilon_{i}$ is the incident medium permittivity, and $f(z)$ is the filling fraction occupied by the pyramid at $z$, which is equal to the ratio between the cross-sectional area of the pyramid and the area of the unit cell of the lattice. Other components of $\hat{\varepsilon}(z)$ depend on the pyramid base shape. For example, $\varepsilon_{x}(z)=\varepsilon_{y}(z)$ for some symmetrical cases.

For a circle base, the Maxwell-Garnett [20,21] expression can be used:

$$
\varepsilon_{x}=\varepsilon_{y}=\varepsilon_{i}+2 f \varepsilon_{i} \frac{\varepsilon_{s}-\varepsilon_{i}}{\varepsilon_{s}+\varepsilon_{i}-f\left(\varepsilon_{s}-\varepsilon_{i}\right)} .
$$

For a square shape, Brauer and Bryngdahl [22] proposed the following empirical expression:

$$
\varepsilon_{x}=\varepsilon_{y}=([\bar{n}+2 \hat{n}+2 \stackrel{\mathfrak{d}}{n}] / 5)^{2},
$$

where

$$
\begin{gathered}
\bar{n}=(1-f) \varepsilon_{i}^{1 / 2}+f \varepsilon_{s}^{1 / 2}, \\
\hat{n}^{2}=\left(1-f^{1 / 2}\right) \varepsilon_{i}+f^{1 / 2}\left(\frac{f^{1 / 2}}{\varepsilon_{s}}+\frac{1-f^{1 / 2}}{\varepsilon_{i}}\right)^{-1}, \\
1 / n^{2}=\frac{\left(1-f^{1 / 2}\right)}{\varepsilon_{i}}+\frac{f^{1 / 2}}{f^{1 / 2} \varepsilon_{s}+\left(1-f^{1 / 2}\right) \varepsilon_{i}} .
\end{gathered}
$$

As was shown previously, an increase in the pyramid height $d$ and a decrease in the optical contrast between the incident medium and the texture reduces the reflection $[10,11]$. Some special profiles $f(z)$ were proposed to reduce the reflection as well [10]. In this section, we first estimate the asymptotic behavior of the reflection coefficient with the increasing $d$ and show how it depends on the tiling of the pyramid bases.

In the case of complete tiling, the filling fraction at the top of the pyramid is $f(0)=0$ and $f(d)=1$ at the base of the pyramid; therefore, $\varepsilon(0)=\varepsilon_{i}$ and $\varepsilon(d)=\varepsilon_{s}$. For the normal incidence case, if the reflectivity $\rho$ is low, the expression derived by Franceschetti is applicable [23]:

$$
\rho=-\int_{0}^{d} \frac{1}{2 \tilde{n}} \frac{d \tilde{n}}{d z} \exp \left[-i \frac{4 \pi}{\lambda} \int_{0}^{z} \tilde{n}\left(z^{\prime}\right) \mathrm{d} z^{\prime}\right] \mathrm{d} z,
$$

where $\tilde{n}(z)=\varepsilon^{1 / 2}(z)=F\left(\varepsilon_{i}, \varepsilon_{s}, f(z)\right)$ is the effective refractive index, $F$ is the function defined by the pyramid base shape and the incident wave polarization [e.g., see Eqs. (2) and (3)]. If $\varepsilon_{x}(z)=\varepsilon_{y}(z), F$ does not depend on the incident wave polarization. 
Let us introduce a new integration variable $g=4 \pi$ $\int_{0}^{z} \tilde{n}\left(z^{\prime}\right) \mathrm{d} z^{\prime}$. Under the assumption of infinite differentiability of the function $\tilde{n}$ and correspondingly $h=\frac{1}{2 \tilde{n}} \frac{d \tilde{n}}{d z} \frac{d z}{d g}$, we can integrate Eq. (7) by parts:

$$
\rho=-\int_{0}^{g(d)} h e^{-i g / \lambda} \mathrm{d} g=\left.\sum_{k=1}(-i)^{k} \lambda^{k} h^{(k-1)} e^{-i g / \lambda}\right|_{0} ^{g(d)} .
$$

One can see from Eq. (8) that the polynomial degree of the dependence of the reflectance $R=|\rho|^{2}$ on $\lambda$ is determined by the index $k^{\prime}$ of the first nonzero item in Eq. (8) as $R \sim \lambda^{2 k^{\prime}}$. Note that, for the effective medium limit, the only scaling parameter of the system is $d / \lambda$, so $R \sim(d / \lambda)^{-2 k^{\prime}}$ at different $d$ and $\lambda$. For the linear profile case, $f(z)=z / d$, the first term in Eq. (8) is nonzero due to $h \sim \frac{d \tilde{n}}{d z} \sim \frac{d f}{d z} \neq 0$; therefore, $R \sim(d / \lambda)^{-2}$.

One can influence the rate of the reflectance reduction with the growth of $d / \lambda$ using a special profile $f(z)$. For example, $f(z)$ can be chosen as polynomial of the degree of $\left(2 k^{\prime}-1\right)$ and $f^{(i)}(0)=f^{(i)}(d)=0$ for $i<k^{\prime}$, where $f^{(i)}$ is the $i$ derivative of $f$. This leads to $h^{(i)}(0)=h^{(i)}(d)=0$ for $i<k^{\prime}-1$; therefore, the first $k^{\prime}-1$ items in Eq. () are zero and $R \sim(d / \lambda)^{-2 k^{\prime}}$. In particular for the profiles proposed by Southwell [10], $f(z)=3 z^{2}-2 z^{3}$ and $f(z)=10 z^{3}-15 z^{4}+6 z^{5} \quad$ in these expressions, $d=1$ ), we obtain $R \sim(d / \lambda)^{-4}$ and $R \sim(d / \lambda)^{-6}$ correspondingly.

Let us find a profile characterized by zero derivatives of all orders at the points 0 and $d: f^{(i)}(0)=f^{(i)}(d)=0, \forall i>0$. Without any restriction, assume that $d=1$. Consider first the infinitely differentiable function $e^{-z^{-1}(1-z)^{-1}}$, which is zero with all its derivatives at $z=0$ and $z=1$. After its integration, we get a monotone function increasing from 0 to $1 f(z)=$ $C \int_{0}^{z} e^{-\zeta^{-1}(1-\zeta)^{-1}} \mathrm{~d} \zeta$, where the value of $C$ is chosen to ensure $f(1)=1$. Using this "integral" profile leads to the exponential decrease of the reflectance with the growth of $d / \lambda$ due to $f^{(i)}(0)=f^{(i)}(d)=0, \forall i>0$. The shapes of the discussed profiles are presented in Fig. 3.

We calculated dependence of the reflectance on $d / \lambda$ for gradient index layers corresponding to closely packed square pyramids with flat-sided $\left(f(z)=z^{2}, k^{\prime}=1\right.$ because, for flatsided pyramids, the width depends linearly on the height and the filling fraction is proportional to the width squared), cubic $\left(f(z)=3 z^{2}-2 z^{3}, k^{\prime}=2\right)$, and quintic $\left(f(z)=10 z^{3}\right.$ $15 z^{4}+6 z^{5}, k^{\prime}=3$ ) profiles. We used Eq. (3) for the effective refractive index of square pyramids. Calculations were performed using the $2 \times 2$ matrix technique described in [1]. We obtained $R \sim(d / \lambda)^{-2}, R \sim(d / \lambda)^{-4}$, and $R \sim(d / \lambda)^{-6}$ for the cases considered here (Fig. $\underline{4}$, left).

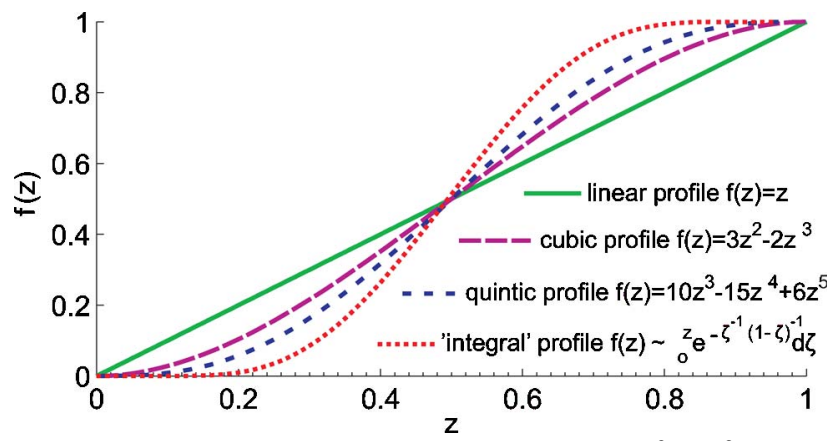

Fig. 3. (Color online) Profiles $f(z)=z, f(z)=3 z^{2}-2 z^{3}, f(z)=$ $10 z^{3}-15 z^{4}+6 z^{5}$, and $f(z)=C \int_{0}^{z} e^{-\zeta^{-1}(1-\zeta)^{-1}} \mathrm{~d} \zeta$.
We calculated the reflectance for the gradient index film corresponding to a single periodic grating with the integral profile. One can see that using this profile leads to an exponential decrease of the reflection with the growth of $d / \lambda$ (Fig. 4, right).

At the incomplete tiling case, there are gaps between pyramid bases: $0<f(d)<1$ (see cones in Fig. 1), causing a discontinuity of the permittivity $\varepsilon(z)$ at the pyramid bases $z=d: \varepsilon(d) \neq \varepsilon_{i}$. Because of this fact, by increasing $d / \lambda$, the reflectance tends to a constant value equal to the reflectance between the media with $\varepsilon(d)$ and $\varepsilon_{i}$. To demonstrate this, we calculated the reflectance for the gradient index film corresponding to cones closely packed in the triangular lattice (Fig. 4, right). We used the Maxwell-Garnett expression [Eq. (2)] for the effective refractive index of cones.

We performed FDTD calculations for pyramidally textured surfaces corresponding to the gradient index films considered here at a wavelength larger than the pyramid base size: $4<\lambda<\infty, \Lambda=1, d=16$ (FDTD mesh step $\delta x=0.01$ ). Calculations were performed using the subpixel smoothing method $[24,25]$, which allowed us to increase the accuracy compared to the usual staircase model of the pyramids. One can see from Fig. 4 that the FDTD results for pyramids and the results for a layer with gradually changing refractive index are in good agreement. However, a deviation of the FDTD results from the effective medium theory is still seen at large $d / \lambda$ for pyramids with quintic profiles $\left(k^{\prime}=3\right)$ and gratings with the integral profile. It may be explained by the insufficient mesh resolution to represent the pointed pyramid tips, characterizing the selected profiles.

\section{SHORT WAVELENGTH LIMIT}

In the short wavelength limit $\Lambda \gg \lambda$, the optical properties of textured surfaces do not depend on the wavelength $\lambda$ and are defined by geometry only. The ray tracing technique (e.g., see [14]) is widely used for their numerical modeling.

We calculated the reflection for closely packed triangular, hexagonal, and square pyramids (complete tiling) and cones (incomplete tiling) for different values of $d / \Lambda$ using this technique (Fig. 5). We obtained exponential decrease of the
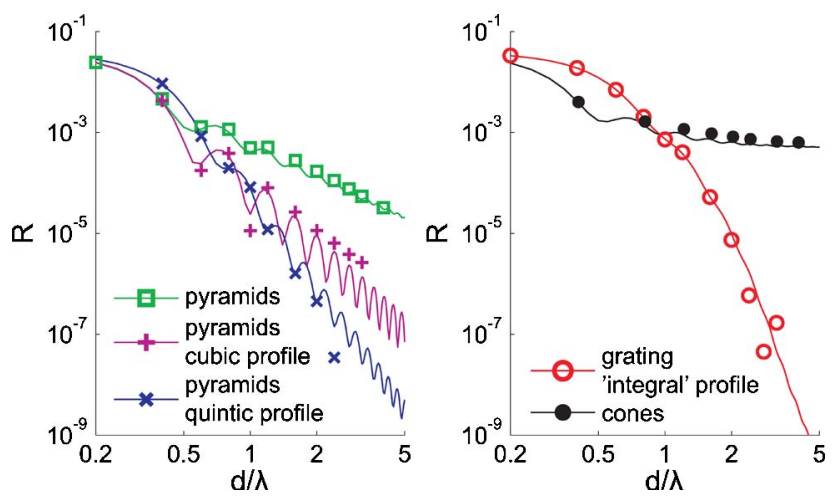

Fig. 4. (Color online) Curves are the reflectance from a graded index film with the optical properties corresponding to square pyramids with flat-sided, cubic, and quintic profiles closely packed in the square lattice (left side); grating with the integral profile $f(z)=$ $C \int_{0}^{z} e^{-\zeta^{-1}(d-\zeta)^{-1}} \mathrm{~d} \zeta, f(d)=1$, TE case; cones closely packed in the triangular lattice (right side). For the effective dielectric permittivity of square pyramids and cones, Eqs. (2) and (3) were used. The FDTD calculations (points) were performed for the corresponding pyramids with $\Lambda=1, d=16$, and $4<\lambda<\infty$ (FDTD mesh step $\delta x=0.01$ ). 
reflection with the growth $d / \Lambda$ for the complete tiling case. For the incomplete tiling case, the reflection tends to a constant value passing a local minimum while $d / \Lambda$ increases. In the following, we give our explanation of these results.

According to [26], we introduce the following ray classification: (a) incident rays; (b) reflected rays formed by incident rays after their reflection from the texture, which revert back into the incident medium after some number $M$ of consecutive reflections; (c) refracted rays formed by incident or reflected rays after they get into the texture; and (d) secondary rays formed by refracted rays if they leave the texture (Fig. 6). Only reflected and secondary rays make contributions to the total reflection $R=R_{\text {refl }}+R_{\text {sec}}$. Here, the partial reflections are defined as the total reflected intensity of the corresponding ray type divided by the incident light intensity. Consider these partial contributions for the complete tiling case. Each incident ray consists of two subrays with parallel or perpendicular polarization relative to the plane of incidence (this plane is defined by the ray and the normal to the pyramid side at the point the ray moves onto it).

At the complete tiling case, bases touch each other at every point of their perimeters. Therefore, the incidence plane does not change, while a ray consecutively reflects between neighbouring sides [Fig. $7(\mathrm{a})$ ]. Thus, the total reflection is $R_{\text {refl }}=$ $\left(R_{\text {refl, } \|}+R_{\text {refl, } \perp}\right) / 2$. We assume that the rays with parallel and perpendicular polarizations make equal contributions to the total incident light intensity (which is achieved in case of uniform illumination).

Let us estimate $R_{\text {refl, } \perp}$. As was shown in [26], during each $m$ th reflection, the ray comes onto the pyramid side at the angle $|\pi / 2-(2 m-1) \beta|$, where $\beta$ is half of the angle between sides of the neighboring pyramids (Fig. 1). Therefore, after $m$ reflections, the ray intensity is multiplied by $R_{\perp}(\mid \pi / 2-$ $(2 m-1) \beta \mid)$, where $R_{\perp}(\phi)$ is Fresnel reflection coefficient for the incidence angle $\phi$ and perpendicular polarization. According to [26], the number of reflections required for the ray to finally revert back into the incident medium is equal to $M$ or $M+1$, where

$$
M \leq \pi /(2 \beta) \leq M+1
$$

Therefore,

$$
R_{\mathrm{refl}, \perp} \approx \prod_{m=1}^{M} R_{\perp}(|\pi / 2-(2 m-1) \beta|)
$$

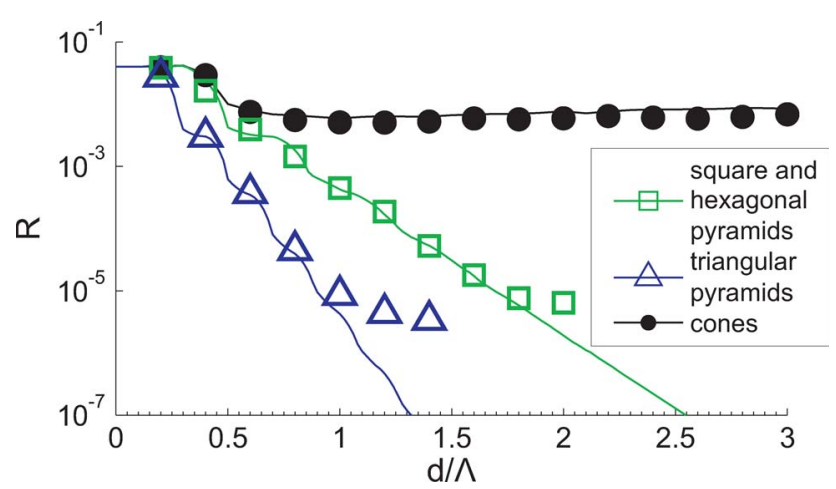

Fig. 5. (Color online) Curves are reflectance from different closely packed structures in the geometric optics limit. FDTD calculations (points) are performed for $\Lambda / \lambda=15$.

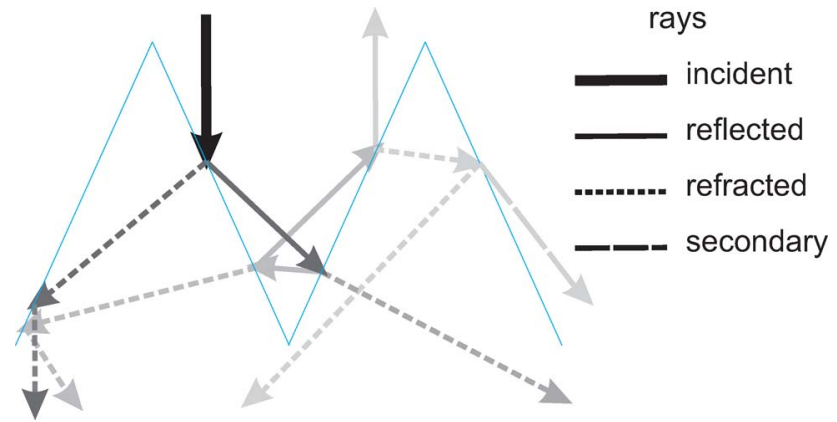

Fig. 6. (Color online) Rays propagated in the texture (the ray tracing simulation). Gray color intensity corresponds to the intensity of the rays.

Taking the logarithm of this expression and assuming that $\beta$ is small and $M$ is large, we obtain

$$
\begin{aligned}
\ln R_{\mathrm{refl}, \perp} & \approx \sum_{m=1}^{M} \ln R_{\perp}(|\pi / 2-(2 m-1) \beta|) \approx \\
& \approx \frac{2 M}{\pi} \int_{0}^{\pi / 2} \ln R_{\perp}(\phi) \mathrm{d} \phi \approx \frac{1}{\beta} \int_{0}^{\pi / 2} \ln R_{\perp}(\phi) \mathrm{d} \phi \\
& =-C \beta^{-1},
\end{aligned}
$$

where $C>0$ since $R_{\perp}(\phi) \leq 1$ and $\ln R_{\perp}(\phi) \leq 0$. Since $\beta$ is small, $\beta=\arctan \frac{L}{d} \approx \frac{L}{d}$, where $L$ is the distance between the base side and its center (Fig. 1). As a result, we obtain

$$
R_{\text {refl, } \perp} \approx \exp \left(-C \frac{d}{L}\right) .
$$

$R_{\|}(\phi) \leq R_{\perp}(\phi)$ for any $\phi$, therefore, $R_{\text {refl, } \|} \leq R_{\text {refl, } \perp \text { (for some }}$ values of $\beta, R_{\text {refl, } \perp}$ can be zero since, after some $m$ th reflection, a ray can move onto the pyramid side under the Brewster's angle). Therefore,

$$
R_{\text {refl }} \approx \exp \left(-C \frac{d}{L}\right) .
$$

According to our calculations, secondary rays make a small contribution to the reflection $R_{\text {sec }} \approx R_{\text {refl }}$, which can be explained by the following considerations. First, pyramids deflect secondary rays downward since $n_{s}>n_{i}$, preventing them from reverting back to the incident medium [Fig. 7(b)].

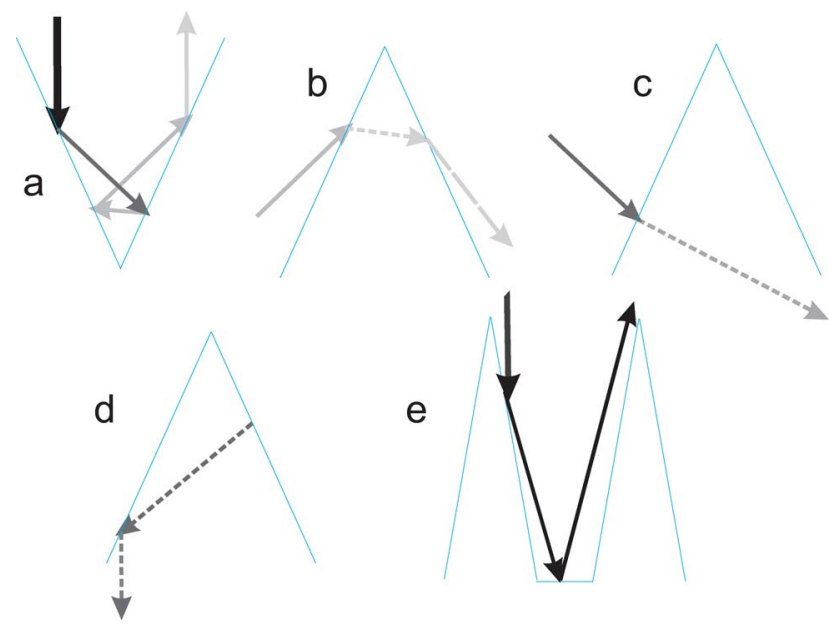

Fig. 7. (Color online) Rays propagated in the texture. 
Second, refracted rays can transmit to the substrate directly [Fig. 7(c)] or move onto the inner pyramid side under the total internal reflection angle [Fig. 7(d)]. This prevents them from forming secondary rays. Therefore,

$$
R \approx \exp \left(-C \frac{d}{L}\right)
$$

in agreement with the numerical results in Fig. 5 , since $L$ depends linearly on $\Lambda$ (Fig. 1 ).

Note that results for hexagonal and square pyramids are almost identical since their $L$ is equal at fixed $\Lambda$ (Fig. 1). The curve tangent for square and triangular pyramids in Fig. 5 is approximately different by a factor of $\sqrt{3}$. This is because $\bar{L}$ for a triangular pyramid is $\sqrt{3}$ times smaller than for a square pyramid at fixed $\Lambda$ (Fig. 1 ).

In the case of incomplete tiling (cones), the reflectance tends to the constant value with the growth of $d / \Lambda$ passing over a local minimum. It can be explained by the following considerations. While $d / \Lambda \rightarrow \infty$, normal rays remain almost parallel to the scatterer surface after the first reflection and some of them go to the gap between the bases not reaching the neighboring scatterer. Afterward, they are directly reflected back into the incident medium [Fig. 7(e)]. For the cones case, almost all incident rays behave in this way; therefore, while $d / \Lambda \rightarrow \infty$, the reflectance tends to the substrate reflectance value.

We performed FDTD calculations for the structures discussed here at $\Lambda / \lambda=15$. Since the FDTD mesh step should be at least 10 times smaller than the wavelength, calculations required large FDTD mesh sizes and were performed on a parallel computer (i.e., calculations with the mesh $150 \times 150 \times$ 300 were performed using 160 processing cores). FDTD and ray tracing results are in good agreement with each other (Fig. 5). The difference between them appearing by increasing $d / \Lambda$ is due to the fact that the value of $\Lambda / \lambda$ used in FDTD becomes insufficiently large for light diffraction effects to be neglected.

\section{FDTD RESULTS FOR ALL TEXTURE SIZE-TO-WAVELENGTH RATIOS}

In this section, we perform a numerical parameter sweep in order to find the global reflection minimum with respect to texture geometrical parameters. We perform calculations using the FDTD method, which is valid for all texture sizeto-wavelength ratios, including effective medium and geometric optics limits.

We use a standard scheme of the FDTD calculation in which propagation of a plane wave impulse through the structure is modeled. During the numerical experiment, the amplitude of the reflected wave is recorded, and then it is transformed to the frequency representation and normalized by the incident spectrum. Finally, we obtain the reflection at some wavelength range corresponding to the simulated temporal width of the incident impulse $w$.

We perform calculations for a fixed texture period value $\Lambda=1$ and a set of height values $0<d<5$. We perform three numerical experiments with different temporal widths of the incident impulse $w=0.01,0.1,1$ for each geometry, which allows us to cover a large total wavelength span because different values of $w$ cover different wavelength ranges. Since,

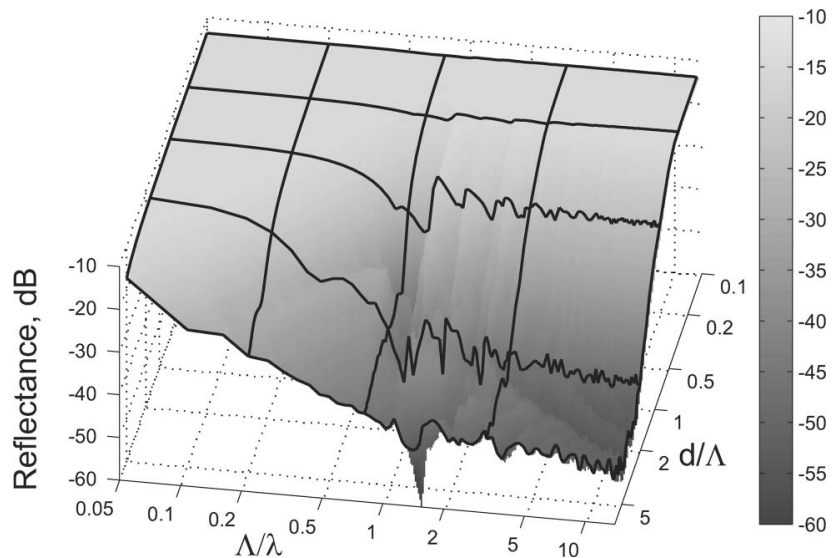

Fig. 8. Reflectance from closely packed pyramids with square bases as a function of $\Lambda / \lambda$ and $d / \Lambda$ (FDTD results).

in our nondispersive case, Maxwell's equations are scaling invariant, we rescale the results for the corresponding $d / \Lambda$ and frequency as if they were obtained at variable $\Lambda / \lambda$.

Consider first the complete tiling case using the results for square pyramids (Fig. 8). Let us fix some value $d / \Lambda$ and follow the reflectance $R$ behavior while $\Lambda / \lambda$ increases (see corresponding black curves at Fig. 8). By the increase of $\Lambda / \lambda$ from 0 to 1 , the reflectance decreases, achieving local minimum at $\Lambda / \lambda \sim 1$. This can be explained by the fact that the effective dielectric permittivity does not depend on the $\Lambda / \lambda$ at zeroth approximation and the reflectance of corresponding gradient index film decreases while $d / \lambda=(d / \Lambda)(\Lambda / \lambda)$ increases. At $\Lambda / \lambda \geq 1$, the reflection decreases further passing local minima corresponding to the values of $\Lambda / \lambda$ at which the next diffraction orders appear. However, these reflectance oscillations become smaller at greater $\Lambda / \lambda$ while the curve approaches the geometric optics limit.

Note that by greater fixed $d / \Lambda$ the curve reaches this limit at greater $\Lambda$ since there are more reflections required for a ray to leave the texture according to Eq. (9). Most of these reflections are near pyramid bases and the path covered by a ray between consecutive reflections becomes small. If $\Lambda / \lambda$ is insufficiently large, then this path is of the order of the wavelength. This makes the geometric optics approximation invalid and increases the effective $\Lambda$ values for which diffraction effects are still important. In the case of incomplete tiling, the minimal reflectance is achieved at $\Lambda$ of the order of the

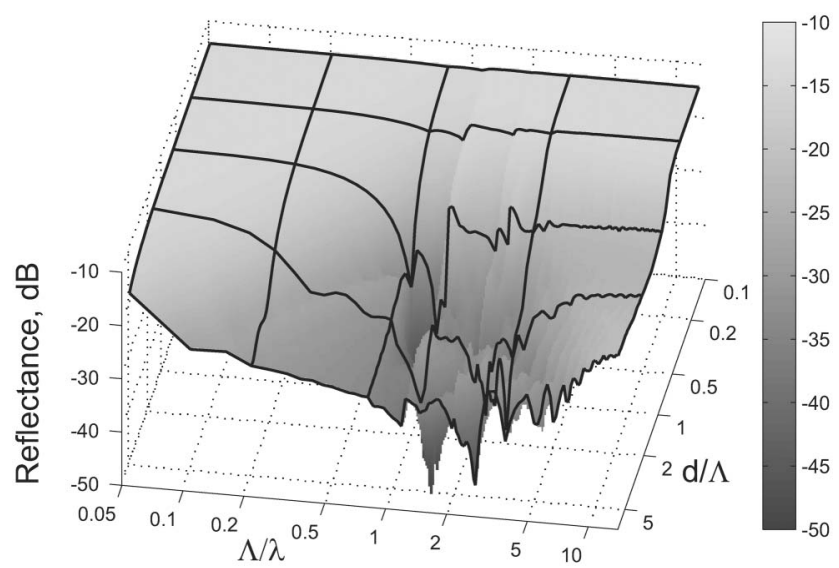

Fig. 9. Reflectance from cones closely packed in the triangular lattice as a function of $\Lambda / \lambda$ and $d / \Lambda$ (FDTD results). 

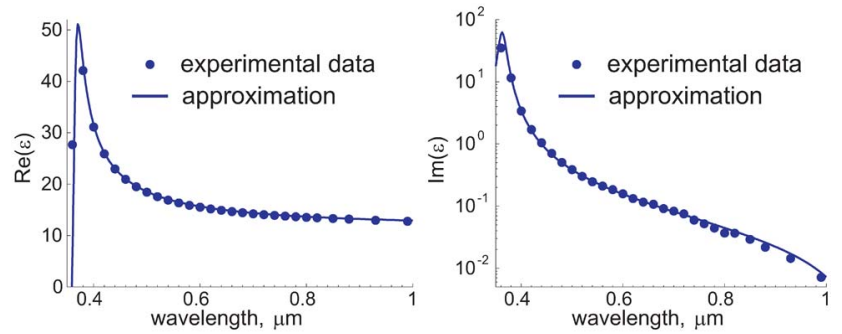

Fig. 10. (Color online) Real and imaginary components of the silicon dielectric permittivity: comparison of the experimental data (dots) with approximation by three Lorentz terms (curves).

wavelength (see results for cones closely packed in the triangular lattice in Fig. 9).

We come to a conclusion that the optimal texture size is determined by the character of the pyramid tiling. In the complete tiling case, the lowest reflection is reached by $\Lambda / \lambda \rightarrow \infty$ for a fixed ratio $d / \Lambda$. This is in agreement with the fact that the rate of the reflection reduction with increasing $d$ and fixed $\Lambda$ and $\lambda$ is exponential in the geometrical optics limit and is thus higher than the polynomial rate in the effective medium limit. At the same time, small reflectance can be achieved at $\Lambda$ of the order of the wavelength. In the incomplete tiling case, the optimal value $\Lambda$ is of the wavelength order. This is also in agreement with the limiting approximations considered above.

Note that high-precision fabrication of completely tiled mactotextured surfaces is a complicated technological task. Fabrication of textured surfaces with periodicity of the order of some hundred nanometers by lithographic technique or etching [7] seems to be more advantageous. A possibility of achieving very small reflection values for texture sizes of the wavelength order is the evidence of this texture efficiency for the visible range.

\section{OPTIMIZATION OF ANTIREFLECTIVE SURFACE OF SOLAR CELLS}

In this section, we consider silicon antireflective textured surfaces used for solar cell efficiency enhancement. As follows from Section $\underline{4}$, at the incomplete tiling case, the optimal scatterer size should be of the order of the wavelength to ensure the minimal reflectance for pure dielectrics. In this section, we demonstrate the validity of this conclusion numerically for silicon-textured surfaces, also taking into account the dispersion in the dielectric permittivity of silicon.

Experimental data on the silicon dielectric permittivity $\varepsilon(\omega)$ were taken from [27]. Usually the frequency dependence of the dielectric permittivity can be assigned in FDTD by consid-
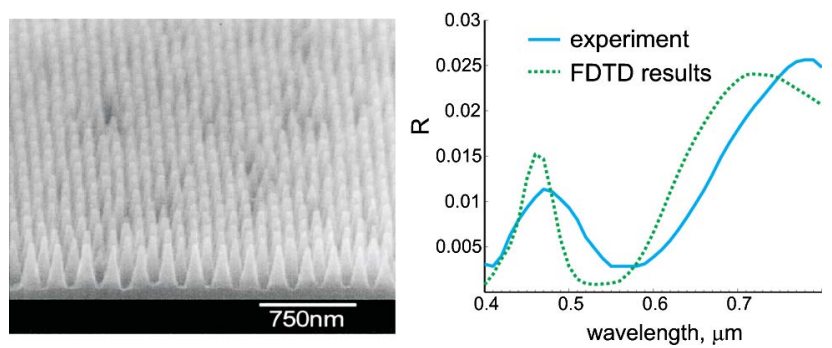

Fig. 11. (Color online) Image of the textured surface investigated in [3] (left). Comparison of the corresponding experimental data with FDTD calculations (right).
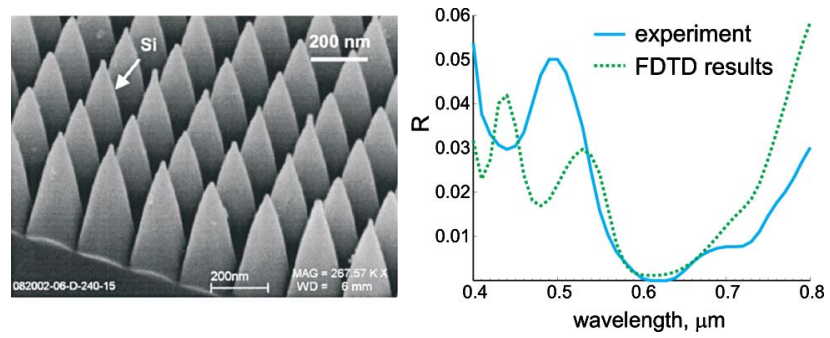

Fig. 12. (Color online) Image of the textured surface investigated in [6] (left). Comparison of the corresponding experimental data with FDTD calculations (right).

ering a Drude-Lorentz approximation. We find an approximation of the silicon permittivity by three Lorentz terms:

$$
\varepsilon(\omega)=\varepsilon_{\infty}+\sum_{p=1}^{3} \frac{\Delta \varepsilon_{p} \omega_{p}^{2}}{\omega_{p}^{2}-2 i \omega \gamma_{p}-\omega^{2}},
$$

with the following parameter values $\left(\omega_{p}\right.$ and $\gamma_{p}$ are in $\left.1 / \mu \mathrm{m}\right)$ : $\varepsilon_{\infty}=1, \Delta \varepsilon_{1}=8, \Delta \varepsilon_{2}=2.85, \Delta \varepsilon_{3}=-0.107, \omega_{1}=3.64, \omega_{2}=$ $2.76, \omega_{3}=1.73, \gamma_{1}=0, \gamma_{2}=0.063$, and $\gamma_{3}=2.5$. There is excellent agreement between tabular frequency dependence and our approximation in the visible range (Fig. 10).

We have simulated the behavior of a silicon surface covered by cones with diameter $\Lambda$ and height $d$. To check the relevance of this geometry to describe experimental structures, the experimental data taken from [3] $(\Lambda=0.15 \mu \mathrm{m}, d=0.35 \mu \mathrm{m}$, triangular lattice) and [6] $(\Lambda=0.2 \mu \mathrm{m}, d=0.52 \mu \mathrm{m}$, square lattice) were compared with the results calculated numerically using FDTD. In these two works, the fabrication of gratings formed by cone-shaped nanoscale silicon pillars is considered (see images in Figs. 11 and 12) and their reflectivity is measured. In both works, the filling fraction at the base of the cones $f(d)$, defining the gap between them, is not specified and should be implied from the images. We calculated the reflectance of the corresponding structures with different filling fraction values and the best agreement with the experimental data was found to be $f(d)=0.9$ for the first structure and $f(d)=0.55$ for the second one (Figs. 11 and 12). The FDTD results presented here as well as all FDTD results in this section were obtained using mesh steps $\delta x=10 \mathrm{~nm}$ (using smaller mesh steps does not influence the FDTD results).

Now we switch to the numerical results obtained for cones with different values of height $d$ and diameter $\Lambda$. We assume that the cone bases are closely packed in the triangular lattice (filling fraction is $f(d)=\pi /(2 \sqrt{3})$ ).
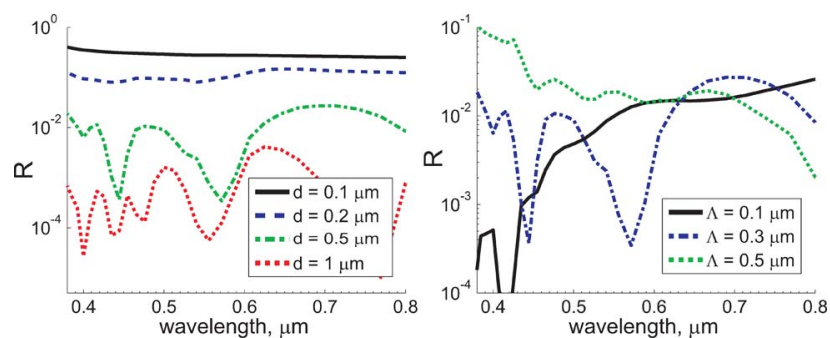

Fig. 13. (Color online) Reflectance for cones with diameter $\Lambda=$ $0.3 \mu \mathrm{m}$ for different heights $d$ (left). Reflectance for cones with height $d=0.5 \mu \mathrm{m}$ for different diameters $\Lambda$ (right). Cones are arranged in the closely packed triangular lattice. One can see that the wavelength range in which the reflection reaches a minimum is shifted to the left with decreasing $\Lambda$. The results are obtained by FDTD. 


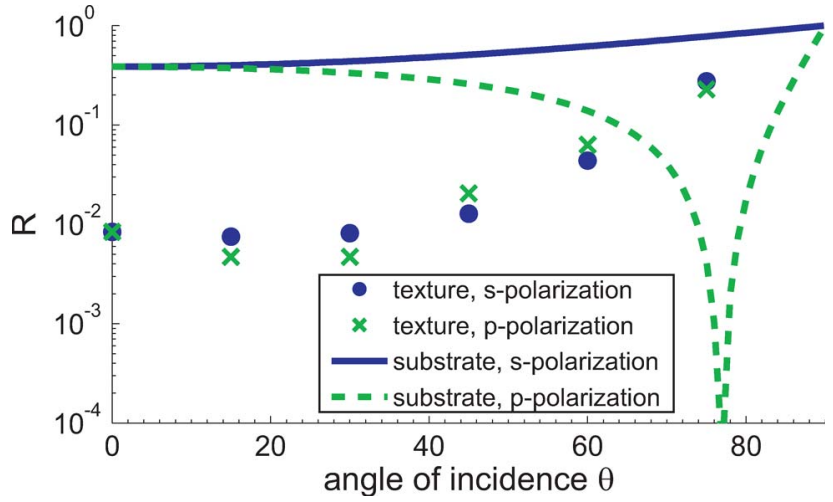

Fig. 14. (Color online) Reflectance for bare substrate and cones with $\Lambda=0.3 \mu \mathrm{m}, d=0.5 \mu \mathrm{m}$ arranged in the closely packed triangular lattice for different angles of incidence and the wavelength $\lambda=0.5 \mu \mathrm{m}$. Results were calculated by FDTD. For oblique incidence calculation, the iterative FDTD technique [28] is applied.

We found that the reflectance $R$ decreases with the growth of the cone height $d$ (see the FDTD results for cones of diameter $\Lambda=0.3 \mu \mathrm{m}$ in Fig. 13). We also found that the minimal reflectance at fixed $d$ is achieved at diameter $\Lambda$ of the order of the average wavelength between the free space and silicon (Fig. 13). The wavelength in silicon is by a factor $n=\operatorname{Re}(\sqrt{\varepsilon})$ less than the wavelength in free space (one can see from Fig. 10 that this ratio is 4 for the visible range). Therefore, the optimal size $\Lambda$ for the visible range $(380-760 \mathrm{~nm})$ is about several hundred nanometers.

Finally, the minimal reflectance $R$ is achievable at the diameter size of the order of the wavelength and when the height is made as large as possible. At the same time, $d \approx 0.5 \mu \mathrm{m}$ is already sufficient for the low reflectance value $(\sim 1 \%)$.

This conclusion remains valid for the oblique incidence case as well (Fig. 14). In this case, the reflectance increases with the growth of the incidence angle $\theta$. When a p-polarized wave is incident at an angle close to the Brewster angle, the reflection of the substrate appears lower than of the textured surfaces. It can be explained by the fact that, in the latter case, the wave ceases to be p-polarized with respect to the side cone surface. However, the reflection coefficient integrated over all angles is still smaller for the textured surface than the one of the substrate.

\section{CONCLUSION}

In this paper, we consider antireflective properties of textured surfaces at the whole range of their size relative to the wavelength including long and short wavelength limits. We use the FDTD method for direct numerical solution of Maxwell's equations. Also, we apply effective medium and geometric optics approximations for the long and short wavelength limits correspondingly.

We found asymptotic behavior of the reflectance with the change of geometric parameters of scatterers composing the texture for both limiting cases. We established that key factor influencing the optimal scatterer size is the character of the tiling substrate of the scatterers. In particular, we showed that the textured surfaces with the periodicity of the order of several hundred nanometers are highly efficient for the visible range. In future work, we plan to extend our study to the oblique light incidence with the help of the iterative FDTD technique [28].

\section{ACKNOWLEDGMENTS}

This work is supported by the Programs of Fundamental Research of the Presidium of RAS number 13 (coord. Savin G.I.) and the Russian Federal Agency for Science and Innovations (contract 02.523.11.3014). Calculations were performed partially on the cluster MVS-100K (JSCC RAS).

\section{REFERENCES}

1. M. Born and E. Wolf, Principles of Optics (Pergamon, 1980).

2. H. A. Macleod, Thin Film Optical Filter (McGraw-Hill, 1989).

3. Y. Kanamori, M. Sasaki, and K. Hane, "Broadband antireflection gratings fabricated upon silicon substrates," Opt. Lett. 24 1422-1424 (1999).

4. C. G. Bernhard, "Structural and functional adaptation in a visual system," Endeavour 26, 79-84 (1967).

5. P. B. Clapham and M. C. Hutley, "Reduction of lens reflexion by the 'Moth Eye' principle," Nature 244, 281-282 (1973).

6. Z. Yu, H. Gao, W. Wu, H. Ge, and S. Y. Chou, "Fabrication of large area subwavelength anti-reflection structures on $\mathrm{Si}$ using trilayer resist nanoimprint lithography and lift-off," J. Vac. Sci. Technol. B 21, 2874-2877 (2003).

7. Y. F. Huang, S. Chattopadhyay, Y. J. Jen, C. Y. Peng, T. A. Liu, Y. K. Hsu, C. L. Pan, H. C. Lo, C. H. Hsu, Y. H. Chang, C. S. Lee, K. H. Chen, and L. C. Chen, "Improved broadband and quasiomnidirectional anti-reflection properties with biomimetic silicon nanostructures," Nat. Nanotechnol. 2, 770-774 (2007).

8. H. L. Chen, S. Y. Chuang, C. H. Lin, and Y. H. Lin, "Using colloidal lithography to fabricate and optimize sub-wavelength pyramidal and honeycomb structures in solar cells," Opt. Express 15, 14793-14803 (2007).

9. Y. M. Song, S. Y. Bae, J. S. Yu, and Y. T. Lee, "Closely packed and aspect-ratio-controlled antireflection subwavelength gratings on GaAs using a lenslike shape transfer," Opt. Lett. 34, 1702-1704 (2009)

10. W. H. Southwell, "Pyramid-array surface-relief structures producing antireflection index matching on optical surfaces," J. Opt. Soc. Am. A 8, 549-553 (1991).

11. D. H. Raguin and G. M. Morris, "Analysis of antireflectionstructured surfaces with continuous one-dimensional surface profiles," Appl. Opt. 32, 2582-2598 (1993).

12. B. L. Sopori and R. A. Pryor, "Design of antireflection coatings for textured silicon solar cells," Sol. Cells 8, 249-261 (1983).

13. P. Campbell and M. A. Green, "Light trapping properties of pyramidally textured surfaces," J. Appl. Phys. 62, 243-249 (1987).

14. A. A. Abouelsaood, S. A. El-Naggar, and M. Y. Ghannam, "Shape and size dependence of the anti-reflective and light-trapping action of periodic grooves," Prog. Photovolt. Res. Appl. 10, 513-526 (2002).

15. F. Llopis and I. Tobias, "Texture profile and aspect ratio influence on the front reflectance of solar cells," J. Appl. Phys. 100, 124504 (2006).

16. A. Deinega, I. Valuev, B. Potapkin, and Y. Lozovik, "Antireflective properties of pyramidally textured surfaces," Opt. Lett. 35, 106-108 (2010).

17. A. Taflove and S. H. Hagness, Computational Electrodynamics: the Finite Difference Time-Domain Method (Artech House, 2005).

18. A. Deinega and I. Valuev, "Long-time behavior of PML absorbing boundaries for layered periodic structures," Comput. Phys. Commun. 182, 149-151 (2011).

19. Electromagnetic Template Library, http://fdtd. kintechlab. com

20. T. C. Choy, Effective Medium Theory: Principles and Applica tions (Clarendon, 1999).

21. F. Wu and K. W. Whites, "Computation of static effective permittivity for a multiphase lattice of cylinders," Electromagnetics 21, 97-114 (2001).

22. R. Bräuer and O. Bryngdahl, "Design of antireflection gratings with approximate and rigorous methods," Appl. Opt. 33, 7875-7882 (1994).

23. G. Franceschetti, "Scattering from plane layered media," IEEE Trans. Antennas Propag. 12, 754-763 (1964).

24. A. Farjadpour, D. Roundy, A. Rodriguez, M. Ibanescu, P. Bermel, J. D. Joannopoulos, S. G. Johnson, and G. Burr, "Improving 
accuracy by subpixel smoothing in FDTD," Opt. Lett. 31, 29722974 (2006).

25. A. Deinega and I. Valuev, "Subpixel smoothing for conductive and dispersive media in the FDTD method," Opt. Lett. 32, 3429-3431 (2007).

26. O. Bucci and G. Franceschetti, "Scattering from wedge-tapered absorbers," IEEE Trans. Antennas Propag. 19, 96-104 (1971).
27. M. A. Green and M. Keevers, "Optical properties of intrinsic silicon at $300 \mathrm{~K}$," Prog. Photovoltaics 3, 189-192 (1995).

28. I. Valuev, A. Deinega, and S. Belousov, "Iterative technique for analysis of periodic structures at oblique incidence in the finitedifference time-domain method," Opt. Lett. 33, 1491-1493 (2008). 